\title{
Power Spectral Density Analysis of WLAN based OFDM System
}

\author{
Navdeep Kaur \\ Department of Electronics \\ Technology, \\ Guru Nanak Dev University, \\ Amritsar (Punjab), India.
}

\author{
Hardeep Kaur \\ Department of Electronics \\ Technology, \\ Guru Nanak Dev University, \\ Amritsar (Punjab), India.
}

\author{
Jaipreet Kaur \\ Department of Electronics \\ Technology, \\ Guru Nanak Dev University, \\ Amritsar (Punjab), India.
}

\begin{abstract}
OFDM is a technique used in modern broadband wireless communications systems. OFDM has been adopted for wireless standards such as IEEE 802.11a. In this paper, different Power Spectral Density (PSD) curves of OFDM signal based on WLAN with various pulse shapes are presented. OFDM waveform is analyzed in frequency domain response for different modulation techniques such as BPSK, QPSK, 16-QAM, 64-QAM and different block sizes such as 50 and 2048. Power spectral density increases with increase in levels of modulation. For high power spectral density of transmitter higher levels of QAM modulation techniques are preferred.
\end{abstract}

\section{General Terms}

Orthogonal Frequency Division Multiplexing, Modulation techniques, Wireless LAN, Block Size.

\section{Keywords}

WLAN, OFDM, Power Spectral Density, Power Spectrum, Frequency.

\section{INTRODUCTION}

The main key role in wireless radio technologies is focused on effective bandwidth availability given that the spectrum is limited and this stimulates researchers and engineers to use the spectrum more efficiently [1]. The multicarrier modulation techniques are increasingly used in Wireless Local Area Network (WLAN) environments to provide high data rate transmission. The choice of multicarrier technique Orthogonal Frequency Division Multiplexing (OFDM) is due to its good performance in multipath environments. In an OFDM scheme, a large number of orthogonal, overlapping, narrow band sub channels or subcarriers, transmitted in parallel, divide the available transmission bandwidth into several orthogonal subcarriers and each subcarrier is modulated with modulation technique in same bandwidth. The separation of the subcarriers is theoretically minimal such that there is a very compact spectral utilization. Mobility, flexibility, scalability, cost-effectiveness and rapid deployment are some of the factors driving the proliferation of this technology [2].

\section{OFDM TRANSMISSION}

Orthogonal Frequency Division Multiplexing (OFDM) is an attractive multiplexing technique used for transmitting large amounts of data over radio waves. OFDM has several properties like high spectral efficiency, robustness to channel fading, immune to impulse interference, uniform spectral density, and capacity to handle very strong echoes and less non-linear distortion [3].

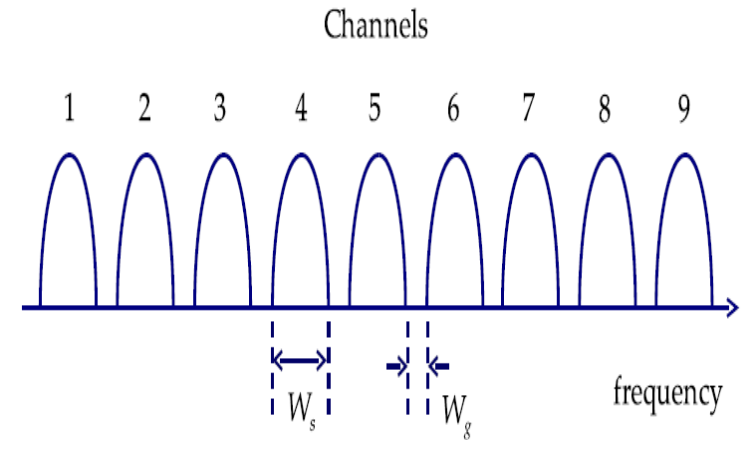

(a) Conventional multicarrier technique

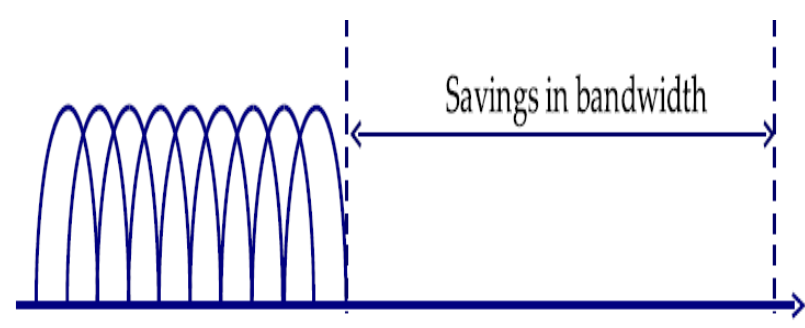

frequency

(b) Orthogonal multicarrier modulation technique

Figure 1: Concept of the OFDM signal: (a) conventional multicarrier technique, and (b) orthogonal multicarrier modulation technique [4].

OFDM is a multiplexing technique that divides an OFDM signal which is a sum of several sinusoids channel with a higher relative data rate into several orthogonal sub-channels with a lower data rate and has become one of the standard choices for high-speed data transmission. A large number of closely spaced orthogonal subcarriers are used to carry data. The data is divided into several parallel streams of channels, one for each subcarrier. Each subcarrier is modulated with a conventional modulation scheme at a low symbol rate, maintaining total data rate similar to the conventional single carrier modulation schemes in the same bandwidth. The carriers are independent of each other even though their spectra overlap. High data rate systems are achieved by using a large number of carriers. OFDM allows for a high spectral efficiency as the carrier power, and modulation scheme can be individually controlled for each carrier. In conventional system up to $50 \%$ of total spectrum is wasted due to extra spacing between channels. This problem overcomes by using wider bandwidth channels [4]. 


\section{Wireless LAN / IEEE 802.11}

The IEEE released the 802.11 specifications in June 1999 . The 802.11 specifications were developed specifically for Wireless Local Area Networks (WLANs) by the IEEE and include four subsets of Ethernet-based protocol standards: $802.11,802.11 \mathrm{a}, 802.11 \mathrm{~b}$, and $802.11 \mathrm{~g}$.

Table 1. Physical layer simulation parameters for IEEE 802.11a [3]

\begin{tabular}{|c|c|}
\hline PARAMETERS & VALUES \\
\hline Channel spacing & $20 \mathrm{MHz}$ \\
\hline IFFT & 64 \\
\hline $\begin{array}{c}\text { Subcarrier } \\
\text { Spacing }\left(\mathrm{F}_{\mathrm{c}}\right)\end{array}$ & $312.5 \mathrm{KHz}(=20 \mathrm{MHz} / 64)$ \\
\hline $\begin{array}{c}\text { Useful symbol } \\
\text { period }\end{array}$ & $3.2 \mu \mathrm{sec}$ \\
\hline $\begin{array}{c}\text { Modulation schemes } \\
\text { BoSK, QPSK, } 16-\mathrm{QAM}, \\
64-\mathrm{QAM}\end{array}$ & $4 \mu \mathrm{sec}$ \\
\hline $\begin{array}{c}\text { Total symbol period } \\
\text { Used subcarrier } \\
\text { index }\end{array}$ & $\{26$ to $1,+1$ to +26$\}$ \\
\hline
\end{tabular}

OFDM has been considered by many IEEE standard working groups, such as IEEE $802.11 \mathrm{a} / \mathrm{g} / \mathrm{n}$, IEEE $802.15 .3 \mathrm{a}$, and IEEE $802.16 \mathrm{~d} / \mathrm{e}$. 802.11a operates in the 5 to $6 \mathrm{GHz}$ range with data rates commonly in the $6 \mathrm{Mbps}, 12 \mathrm{Mbps}$, or $24 \mathrm{Mbps}$ range. As $802.11 \mathrm{a}$ uses the orthogonal frequency division multiplexing (OFDM) standard, data transfer rates can be as high as $54 \mathrm{Mbps}$ [7]. OFDM breaks up fast serial information signals into several slower sub signals that are transferred at the same time via different frequencies, providing more resistance to radio frequency interference. The modulation parameters of OFDM for IEEE 802.11a are listed in Table 1.

\section{IMPLEMENTATION MODEL OF WLAN}

Before transmitting information data, there are different modulations schemes are used. The data is modulated in a baseband fashion by the IFFT. Then the data is transmitted to the channel. The receiver performs the inverse process of the transmitter [3]. Figure 2 shows the general architecture of a typical OFDM transceiver. In the data transmitter section OFDM signal is generated by firstly choosing the spectrum based on input data and modulation scheme used. Each carrier to be produced is assigned same data to transmit. This spectral representation of the data is converted back into the time domain using an Inverse Fast Fourier Transform (IFFT). The Inverse Fast Fourier Transform (IFFT) is much more computationally efficient, and provides a simple way of ensuring the carrier signals produced are orthogonal.
Figure 2: OFDM Transceiver [5]

OFDM Transmitter

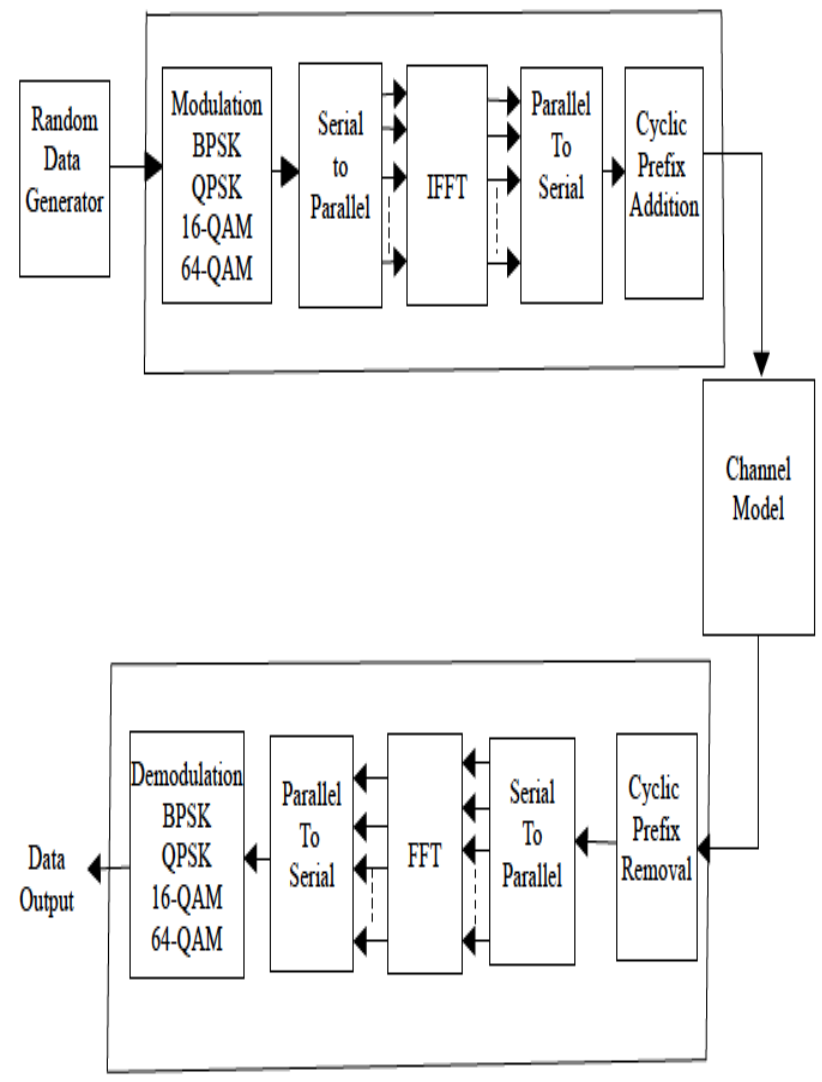

OFDM Receiver

In order to transmit the OFDM signal the calculated time domain signal is then mixed up to the required frequency. The receiver performs the reverse operation of the transmitter, mixing the RF signal to base band for processing, then using Fast Fourier Transform (FFT) to analyze the signal in the frequency domain [5].

\section{CONCEPT OF TRANSMIT POWER SPECTRUM}

In an OFDM signal, the complex values modulating the subcarriers in each symbol period are statistically independent of each other. Each carrier is modulated using Binary Phase Shift Keying (BPSK) or Quaternary Phase Shift Keying (QPSK) or Quadrature Amplitude Modulation (QAM). Instead of separating each of the 52 carriers with a guard band, OFDM overlaps them. OFDM avoids Inter Carrier Interference ICI problem because of its orthogonal property and by precisely controlling the relative frequencies and timing of the carriers. They are also independent of the values modulating any subcarrier in any previous or subsequent symbol period. As a result the power spectrum of the overall signal can be found by summing the power spectra of all individual subcarriers for any symbol period [6].

Power Spectral Density function (PSD) shows the strength of the variations (energy) as a function of frequency. It shows at which frequencies variations are strong and at which frequencies variations are weak. The unit of power spectral density is energy per frequency (width) and energy can be obtained within a specific frequency range by integrating 
Power spectral density within that frequency range. The power spectral density is averaged across $20 \mathrm{MHz}$ bandwidth as specified by WLAN. Number of data symbols that constitute the envelope of power spectrum, spread over frequency bins. Power spectral density depends on levels of modulation as it increases with increase in levels of modulation.

\section{SIMULATIONS AND RESULTS}

The simulations are presented to analyze transmit power spectrum for various digital modulation techniques and block sizes for WLAN standard of OFDM system. Plots between Power spectral density and frequency for digital modulation techniques and bock sizes are plotted and its comparisons are carried out. Simulations are performed on the different types of modulation schemes and block sizes with same parameters. The aim of doing the simulation is to study transmit power spectrum based on WLAN under different modulation techniques and block sizes.

\subsection{Simulation Parameters}

The system proposed in Figure 3 and Figure 4 has been simulated in Matlab. Table 2 lists the main simulation parameters. They are based on the OFDM standard IEEE 802.11a. The data is generated in the form of vector of 2400 bits fixed for all digital modulation simulations. The data is split in the OFDM carriers enhancing spectral efficiency. The extra pilot signal carrier giving information to receiver about communication system is added. The function of adding cyclic prefix is to add some last part of sequence according to guard length and add it with the whole part.

The power spectral density is calculated in units of power per radians per sample. The length $\mathrm{N}$ of the FFT and the values of the input $\mathrm{x}$ determine the length of power spectral density and the range of the corresponding normalized frequencies.

Table 2. Parameters used in simulation

\begin{tabular}{|c|c|}
\hline PARAMETERS & VALUES \\
\hline Total data & 2400 \\
\hline $\begin{array}{c}\text { Number of OFDM } \\
\text { symbols }\end{array}$ & 40 \\
\hline $\begin{array}{c}\text { Number of data } \\
\text { subcarriers }\end{array}$ & 52 \\
\hline $\begin{array}{c}\text { Number of FFT } \\
\text { points }\end{array}$ & 64 \\
\hline $\begin{array}{c}\text { Modulation Scheme } \\
\text { BPSK, QPSK, 16-QAM, } \\
\text { Cyclic Prefix }\end{array}$ & 16 \\
\hline Frequency & $20 \mathrm{MHz}$ \\
\hline Block Size & 50,2048 \\
\hline
\end{tabular}

\subsection{Simulation and Results}

Simulations are performed on the different types of modulation schemes and block sizes with same parameters. The aim of doing the simulation is to study transmit power spectrum based on WLAN under different modulation techniques and block sizes.

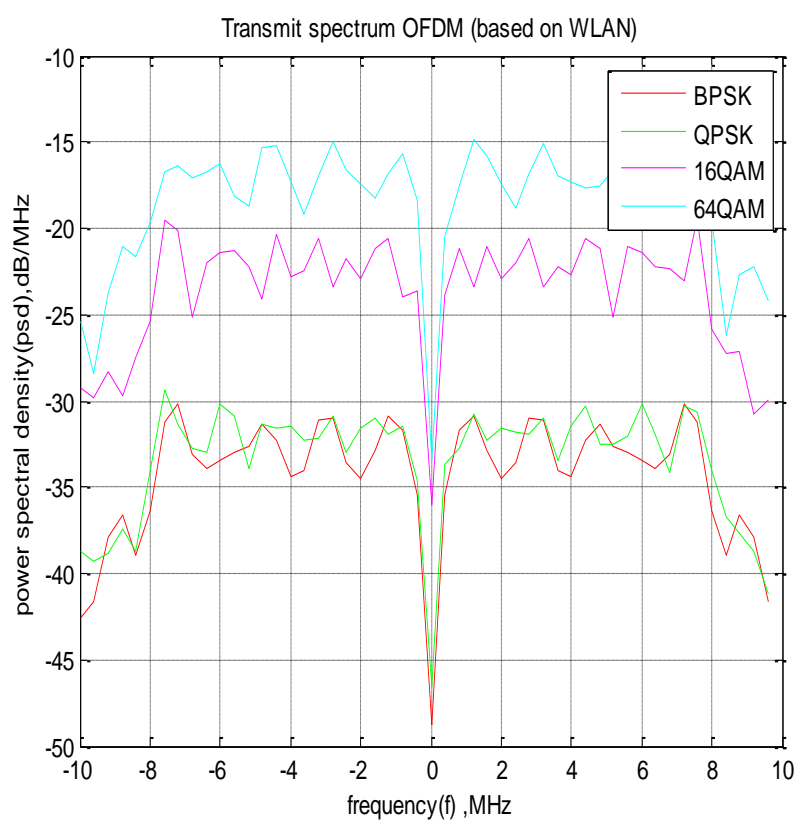

Figure 3: Transmit power spectrum for all digital modulation techniques in WLAN for block size 50

Table 3. Simulated Power Spectral Density (PSD, $\mathrm{dB} / \mathrm{MHz}$ ) with frequency (MHz) for all modulation techniques for block size 50

\begin{tabular}{|c|c|}
\hline $\begin{array}{c}\text { MODULATION } \\
\text { TECHNIQUE }\end{array}$ & $\begin{array}{c}\text { POWER SPECTRAL } \\
\text { DENSITY } \\
\text { (PSD,dB/MHz) }\end{array}$ \\
\hline BPSK & -32.5 \\
\hline QPSK & -32.5 \\
\hline 16-QAM & -22.5 \\
\hline 64-QAM & -17 \\
\hline
\end{tabular}

Figure 3 and Table 3 shows that for all techniques WLAN based OFDM, on fixing block size 50 the Power spectral density increases with increase in number of modulation levels. Power spectral density has constant transmit spectrum within range $-8 \mathrm{MHz}$ to $8 \mathrm{MHz}$. From $-10 \mathrm{MHz}$ to $-8 \mathrm{MHz}$ and for $8 \mathrm{MHz}$ to $10 \mathrm{MHz}$ shows side lobes of transmit spectrum. Power spectral density strengthens within range $-8 \mathrm{MHz}$ to 8 MHz. 


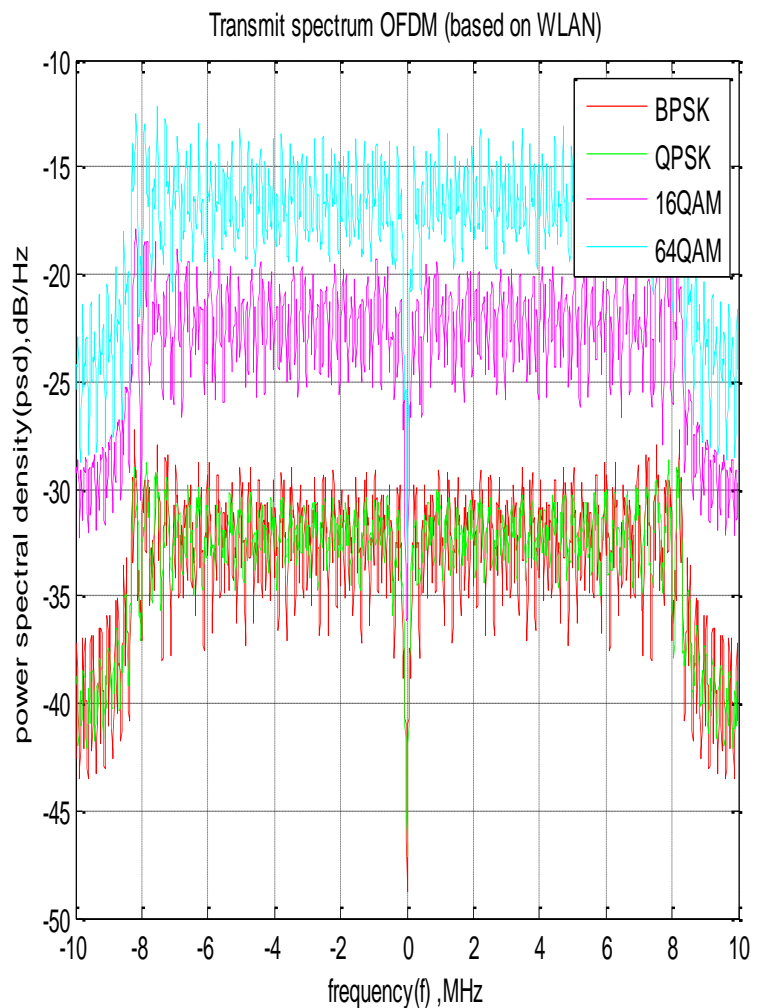

Figure 4: Transmit power spectrum for all digital modulation techniques in WLAN for block size 2048

Figure 4 and Table 4 shows that for all techniques of WLAN based OFDM, on fixing block size 2048 the Power spectral density remain unaffected with increase in block size however Power spectral density increases with increase in number of modulation levels.

Table 4. Simulated Power Spectral Density (PSD,

$\mathrm{dB} / \mathrm{MHz}$ ) with frequency (MHz) for all modulation techniques for block size 2048

\begin{tabular}{|c|c|}
\hline $\begin{array}{c}\text { MODULATION } \\
\text { TECHNIQUE }\end{array}$ & $\begin{array}{c}\text { POWER SPECTRAL } \\
\text { DENSITY(PSD,dB/MHz) }\end{array}$ \\
\hline BPSK & -32.5 \\
\hline QPSK & -32.5 \\
\hline 16-QAM & -22.5 \\
\hline 64-QAM & -17 \\
\hline
\end{tabular}

\section{CONCLUSION}

It can be concluded that Power spectral density depends on the chosen modulation scheme. Power spectral density strengthens with increase in number of modulation levels. In case of BPSK, QPSK modulation techniques the transmitted Power spectral density spectrum superimposed on each other. Power spectral density is better in case of higher levels of QAM modulation techniques. Power spectral density is better in case of 64-QAM modulation techniques than 16-QAM modulation techniques. For all modulation techniques of WLAN based OFDM, it can also be concluded that signal strength is confined within the bandwidth range of $-8 \mathrm{MHz}$ to $8 \mathrm{MHz}$ for different modulation techniques and different block sizes.

\section{REFERENCES}

[1] H A Mohammed, M J N Sibley and P J Mather, "Investigation of Doppler Effects on high mobility OFDM-MIMO systems with the support of High Altitude Platforms", $25^{\text {th }}$ International Congress on Condition Monitoring and Diagnostic Engineering, Journal of Physics: Conference Series 364(2012) 012048, 2012.

[2] Victor P.Gil Jimenez, M. Julia Fernandez-Getino Garcia, "Efficient implementation of complementary Golay sequences for PAR reduction and forward error correction in OFDM-based WLAN systems", AEUInternational Journal of electronics and communications, Vol. 62, Issue 9, pp. 683-694, October 2008.

[3] V. Vijayrangan, DR. (MRS) R. Sukanesh, “An overview of techniques for reducing peak to average power ratio and its selection criteria for Orthogonal Frequency Division Multiplexing radio systems", Journal of Theoretical and applied Information Theory, pp. 25-26, 2005.

[4] Dusan Matiae,"OFDM as a possible modulation technique for multimedia applications", Introduction to OFDM, II edition, pp. 4, October 1998.

[5] Mohammed Safiqul Islam,Gouri Rani Barai,atiq Mahmood, "Performance analysis of different modulation schemes using OFDM techniques in Rayleigh fading channel", International Journal of Fundamental Physical Sciences, Vol.1, No.1, pp. 22-27, March 2011.

[6] Toon van Waterschoot, Vincent Le Nir, Jonathan Duplicy and Marc Moonen, "Analytical Expressions for the Power Spectral Density of CP-OFDM and ZP-OFDM Signals", Draft, December 31,2009.

[7] U.S. Robotics, "Wireless LAN Networking White Paper", pp.1-11. 